\title{
On the Existence of a Periodic Mode in a Nonlinear System
}

\author{
A. S. Fursov ${ }^{1,2,3 *}$, R. P. Mitrev ${ }^{4 * *}$, P. A. Krylov ${ }^{2 * * *}$, and T. S. Todorov ${ }^{4 * * *}$ \\ ${ }^{1}$ Department of Mathematics, School of Science, Hangzhou Dianzi University, Hangzhou, China \\ ${ }^{2}$ Lomonosov Moscow State University, Moscow, 119991 Russia \\ ${ }^{3}$ Kharkevich Institute for Information Transmission Problems, \\ Russian Academy of Sciences, Moscow, 127051 Russia \\ ${ }^{4}$ Technical University, Sofia, 1000 Bulgaria \\ e-mail:*fursov@cs.msu.ru, ${ }^{* *}$ rosenm@tu-sofia.bg, ${ }^{* * *}$ pavel@leftsystem.ru, ${ }^{* * * *}$ tst@tu-sofia.bg
}

Received March 1, 2021; revised May 19, 2021; accepted June 8, 2021

\begin{abstract}
We consider a nonlinear control system with a bang-bang hysteresis control, which is a simplified model of a thermal energy harvester. We obtain conditions on the controller and the system parameters guaranteeing the existence of a periodic mode in the system.
\end{abstract}

DOI: $10.1134 /$ S0012266121080127

\section{INTRODUCTION}

The paper [1] dealt with the nonlinear control system

$$
\begin{gathered}
m \ddot{y}(t)+\beta \dot{y}(t)+k y(t)=\alpha l^{-1} E(T(t))(\Delta-y(t))-m g, \\
\alpha \gamma^{-1} \dot{T}(t)+T(t)=u
\end{gathered}
$$

on the half-line $t \geq 0$ with the initial conditions

$$
y(0)=y_{0}, \quad \dot{y}(0)=0, \quad T(0)=T_{0}
$$

and the bang-bang hysteresis feedback control (Fig. 1)

$$
u=u_{r}(y(t))= \begin{cases}\bar{u} & \text { if } y(t) \leq y_{1} \\ \underline{u} & \text { if } y(t) \geq y_{2} \\ \bar{u} & \text { if } y(\tau) \in\left(y_{1}, y_{2}\right) \text { for all } \tau \in[0, t] \\ \bar{u} & \text { if } y(t) \in\left(y_{1}, y_{2}\right) \text { and there exists an } s \in[0, t) \\ & \text { such that } y(s)=y_{1} \text { and } y(\tau) \in\left(y_{1}, y_{2}\right) \text { for all } \tau \in(s, t] \\ \underline{u} & \text { if } y(t) \in\left(y_{1}, y_{2}\right) \text { and there exists an } s \in[0, t) \\ & \text { such that } y(s)=y_{2} \text { and } y(\tau) \in\left(y_{1}, y_{2}\right) \text { for all } \tau \in(s, t] .\end{cases}
$$

System (1) is a simplified model of a thermal energy harvester [2], where the following notation is used: $y$ is an output variable characterizing the strain of a shape memory material [3] with $0<y_{0}<\Delta ; T$ is the material temperature with $T_{0} \geq 0$; the positive numbers $m, \beta, k, \alpha$, $l, \Delta$, and $\gamma$ are physical parameters of the thermal energy harvester; $g$ is the acceleration due to gravity; $u$ is an output feedback control $(u=u(y))$; and $E$ is the material Young modulus, which is described by a nonlinear characteristic with hysteresis and saturation (see Fig. 2). 


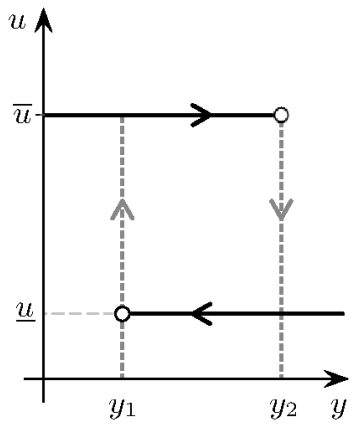

Fig. 1. Bang-bang feedback control.

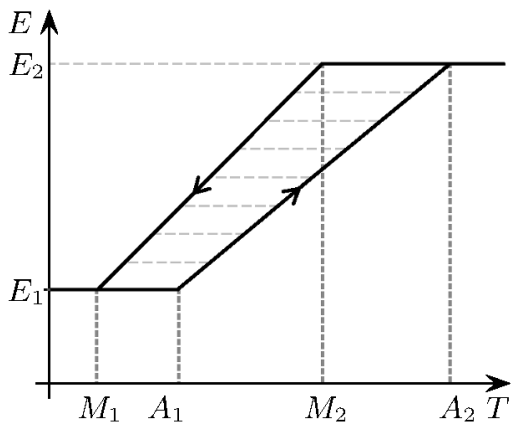

Fig. 2. Possible characteristic $E(T)$ with hysteresis.

The mapping $E(T)$ can be viewed as a set-valued mapping $E: \overline{\mathbb{R}}_{+} \rightarrow \overline{\mathbb{R}}_{+}$,

$$
E(T)= \begin{cases}E_{1} & \text { if } T \leq M_{1} \\
{\left[E_{1}, \frac{T-M_{1}}{M_{2}-M_{1}}\left(E_{2}-E_{1}\right)+E_{1}\right]} & \text { if }\left\{\begin{array}{l}
M_{2}<A_{1} \\
T \in\left(M_{1}, M_{2}\right]
\end{array}\right. \\
{\left[\frac{T-A_{1}}{A_{2}-A_{1}}\left(E_{2}-E_{1}\right)+E_{1}, \frac{T-M_{1}}{M_{2}-M_{1}}\left(E_{2}-E_{1}\right)+E_{1}\right]} & \text { if }\left\{\begin{array}{l}
A_{1} \leq M_{2} \\
T \in\left(A_{1}, M_{2}\right]
\end{array}\right. \\
{\left[\frac{T-A_{1}}{E_{2}-A_{1}}\left(E_{2}-E_{1}\right)+E_{1}, E_{2}\right]} & \text { if }\left\{\begin{array}{l}
M_{2}<A_{1} \\
T \in\left(M_{2}, A_{1}\right]
\end{array}\right. \\
{\left[\frac{T-A_{1}}{A_{2}-A_{1}}\left(E_{2}-E_{1}\right)+E_{1}, E_{2}\right]} & \text { if }\left\{\begin{array}{l}
M_{2}<A_{1} \\
T \in\left(A_{1}, A_{2}\right]
\end{array}\right. \\
E_{2}, & \text { if }\left\{\begin{array}{l}
A_{1} \leq M_{2} \\
T \in\left(M_{2}, A_{2}\right]
\end{array}\right.\end{cases}
$$

Here $A_{1}, A_{2}, M_{1}, M_{2}, E_{1}$, and $E_{2}$ are positive constants determined by the physical properties of the shape memory material. In what follows, we assume that these constants are related by the inequalities

$$
0<A_{1}<A_{2}, \quad 0<M_{1}<M_{2}, \quad M_{1}<A_{1}, \quad M_{2}<A_{2}, \quad 0<E_{1}<E_{2} .
$$

Note that, given a specific continuous function $T(t)$, a single-valued branch of $E(T)$ is selected, which is a continuous function ranging in the interval $\left[E_{1}, E_{2}\right]$ (see [1]).

We assume that the threshold values $y_{1}$ and $y_{2}$ characterizing the control (3) satisfy the condition $0<y_{1}<y_{2}<\Delta$.

Efficiently verifiable conditions on the coefficients and initial values of the state variables in system (1), (2) and on the parameters of the controller (3) ensuring the onset of oscillatory motions [4, pp. 10] in the closed-loop system were obtained in [1], where the following definition of oscillatory motion (oscillatory mode) was used. A solution of system (1), (2), that is, a pair of functions $(y(t), T(t))$ satisfying the system and the initial conditions with the control $u$ given by $(3)$ is called an oscillatory mode if there exist positive constants (mode parameters) $t_{1}^{*}, t_{2}^{*}, y$, and $\bar{y}$ (where $0<t_{1}^{*}<t_{2}^{*}$ and $0<y<\bar{y}<\Delta$ ) such that the following conditions are satisfied:

1. There exists a $t \geq 0$ such that $y(t)=\bar{y}$. 
2. For each $t$ such that $y(t)=\bar{y}$, there exists a $\xi \in\left[t+t_{1}^{*}, t+t_{2}^{*}\right]$ such that $y(\xi)=\underline{y}$ and $y(\tau) \neq \underline{y}$ for all $\tau \in\left(t, t+t_{1}^{*}\right)$.

3. For each $t$ such that $y(t)=\underline{y}$, there exists a $\xi \in\left[t+t_{1}^{*}, t+t_{2}^{*}\right]$ such that $y(\xi)=\bar{y}$ and $y(\tau) \neq \bar{y}$ for all $\tau \in\left(t, t+t_{1}^{*}\right)$.

An analysis of the results in [1] shows that the problem of finding conditions on the parameters of the closed-loop system (1)-(3) guaranteeing the existence of oscillatory modes turns out to be very difficult. However, from the viewpoint of applications, the problem of determining conditions for the existence of a periodic mode in the closed-loop system is more important.

In the present paper, based on the results in [1], we obtain sufficient conditions for the existence of a periodic mode in the closed-loop system (1)-(3).

\section{STATEMENT OF THE PROBLEM}

We write system (1), (2) in the normal Cauchy form

$$
\begin{aligned}
& \dot{x}_{1}(t)=x_{2}(t), \\
& \dot{x}_{2}(t)=-\left(\frac{k}{m}+\frac{\alpha}{l m} E\left(x_{3}(t)\right)\right) x_{1}(t)-\frac{\beta}{m} x_{2}(t)-g+\frac{\alpha}{l m} \Delta E\left(x_{3}(t)\right), \\
& \dot{x}_{3}(t)=-\frac{\gamma}{\alpha}\left(x_{3}(t)-u\right), \\
& x_{1}(0)=y_{0}, \quad x_{2}(0)=0, \quad x_{3}(0)=T_{0},
\end{aligned}
$$

where $x_{1}=y, x_{2}=\dot{y}, x_{3}=T, 0<y_{0}<\Delta$, and $T_{0} \geq 0$.

The corresponding closed-loop system with the controller (3) for $t \geq 0$ has the form

$$
\begin{aligned}
& \dot{x}_{1}(t)=x_{2}(t), \\
& \dot{x}_{2}(t)=-\left(\frac{k}{m}+\frac{\alpha}{l m} E\left(x_{3}(t)\right)\right) x_{1}(t)-\frac{\beta}{m} x_{2}(t)-g+\frac{\alpha}{l m} \Delta E\left(x_{3}(t)\right), \\
& \dot{x}_{3}(t)=-\frac{\gamma}{\alpha}\left(x_{3}(t)-u_{r}\left(x_{1}(t)\right)\right), \\
& x_{1}(0)=y_{0}, \quad x_{2}(0)=0, \quad x_{3}(0)=T_{0},
\end{aligned}
$$

where $0<y_{0}<\Delta$ and $T_{0} \geq 0$; we assume that $y_{0}<y_{1}$ and $T_{0}<M_{1}$. We write the closed-loop system (5) in the vector form

$$
\dot{x}(t)=P\left(x(t), u_{r}\right), \quad x=\left(x_{1}, x_{2}, x_{3}\right)^{\mathrm{T}} .
$$

Now we say that the system

$$
\dot{x}(t)=P(x(t), \bar{u})
$$

is active if the bang-bang relay output is $\bar{u}$ and the system

$$
\dot{x}(t)=P(x(t), \underline{u})
$$

is active if the bang-bang relay output is $\underline{u}$.

Then the solution of the closed-loop system (5) with the initial conditions $x(0)=\left(y_{0}, 0, T_{0}\right)^{\mathrm{T}}$, $y_{0}<y_{1}$, is sought in the class of continuous vector functions $x(t)$ in accordance with the following 
algorithm of active mode alternation:

$$
\dot{x}(t)= \begin{cases}P(x(t), \bar{u}) & \text { if } x_{1}(t) \leq y_{1} \\ P(x(t), \underline{u}) & \text { if } x_{1}(t) \geq y_{2} \\ P(x(t), \bar{u}) & \text { if } x_{1}(t) \in\left(y_{1}, y_{2}\right) \text { and there exists an } s \in[0, t) \\ & \text { such that } x_{1}(s)=y_{1} \text { and } x_{1}(\tau) \in\left(y_{1}, y_{2}\right) \text { for all } \tau \in(s, t] \\ P(x(t), \underline{u}) & \text { if } x_{1}(t) \in\left(y_{1}, y_{2}\right) \text { and there exists an } s \in[0 ; t) \\ & \text { such that } x_{1}(s)=y_{2} \text { and } x_{1}(\tau) \in\left(y_{1}, y_{2}\right) \text { for all } \tau \in(s, t] .\end{cases}
$$

Here the solutions of systems (6) and (7) are matched by continuity at the points of discontinuity of the right-hand side of the system (on the hyperplanes $x_{1}=y_{1}$ and $x_{1}=y_{2}$ ). In what follows, a function $f$ defined on the half-line $t \geq 0$ is said to be $\Theta$-periodic if $f(t+\Theta)=f(t)$ for all $t \geq 0$.

Now let us state a control problem for system (4).

Problem. Find constraints on the number parameters $m, k, \alpha, l, \beta, \Delta$, and $\gamma$ of system (4) as well as on the parameters $y_{1}, y_{2}, \underline{u}$, and $\bar{u}$ of the controller (3) under which there exists a periodic solution (periodic mode) in the closed-loop system (5).

\section{PERIODIC MODE IN SYSTEM WITH PROGRAMMED CONTROL}

We divide the solution of the problem about the existence of a periodic solution in system (5) into several steps. First, we study the existence of a periodic solution of system (4) with the programmed control

$$
u_{p}(t)=\left\{\begin{array}{ll}
\bar{u} & \text { if } t \in\left[q \Theta, q \Theta+\Theta_{1}\right) \\
\underline{u}, & \text { if } t \in\left[q \Theta+\Theta_{1}, q \Theta+\Theta_{1}+\Theta_{2}\right),
\end{array} \quad q=0,1,2, \ldots ; \quad \Theta_{1}+\Theta_{2}=\Theta .\right.
$$

Here $\bar{u}, \underline{u}, \Theta_{1}$, and $\Theta$ are positive parameters of the programmed control.

Now consider the problem of finding control parameter values ensuring the existence of a periodic solution of the closed-loop system (4), (9).

First, note that this control $u(t)$ is a piecewise constant $\Theta$-periodic function. Let us show that the equation

$$
\dot{x}_{3}(t)=-\frac{\gamma}{\alpha}\left(x_{3}(t)-u_{p}(t)\right)
$$

has a $\Theta$-periodic solution for any $\bar{u}, \underline{u}$, and $\Theta>\Theta_{1}>0$. Indeed, the Cauchy formula

$$
x_{3}(t)=x_{3}(s) e^{-\gamma(t-s) / \alpha}+\int_{s}^{t} e^{-\gamma(t-\tau) / \alpha} \frac{\gamma}{\alpha} u(\tau) d \tau
$$

holds for the solutions of Eq. (10) for any $t \geq s \geq 0$. Let $x_{3}(0)=T_{0}$. Since $u_{p}(t) \equiv \bar{u}$ on the interval $\left[0, \Theta_{1}\right)$, it follows from (11) that

$$
\begin{aligned}
x_{3}\left(\Theta_{1}\right) & =T_{0} e^{-\gamma \Theta_{1} / \alpha}+\frac{\gamma}{\alpha} \bar{u} \int_{0}^{\Theta_{1}} e^{-\gamma\left(\Theta_{1}-\tau\right) / \alpha} d \tau \\
& =T_{0} e^{-\gamma \Theta_{1} / \alpha}+\bar{u}\left(1-e^{-\gamma \Theta_{1} / \alpha}\right)=\bar{u}+\left(T_{0}-\bar{u}\right) e^{-\gamma \Theta_{1} / \alpha} .
\end{aligned}
$$

Further, since $u_{p}(t) \equiv \underline{u}$ on the interval $\left[\Theta_{1}, \Theta\right)$, we obtain

$$
\begin{aligned}
x_{3}(\Theta) & =\underline{u}+\left(x_{3}\left(\Theta_{1}\right)-\underline{u}\right) e^{-\gamma \Theta_{2} / \alpha}=\underline{u}+\left(\bar{u}+\left(T_{0}-\bar{u}\right) e^{-\gamma \Theta_{1} / \alpha}-\underline{u}\right) e^{-\gamma \Theta_{2} / \alpha} \\
& =\underline{u}\left(1-e^{-\gamma \Theta_{2} / \alpha}\right)+\bar{u}\left(1-e^{-\gamma \Theta_{1} / \alpha}\right) e^{-\gamma \Theta_{2} / \alpha}+T_{0} e^{-\gamma \Theta / \alpha} .
\end{aligned}
$$

DIFFERENTIAL EQUATIONS Vol. $57 \quad$ No. $8 \quad 2021$ 
In view of the $\Theta$-periodicity of the function $u_{p}(t)$, we conclude that the solution $x_{3}(t)$ is $\Theta$-periodic if and only if $x_{3}(\Theta)=T_{0}$. Then we find the initial condition for the $\Theta$-periodic solution $x_{3}^{\Theta}(t)$ from the representation (13),

$$
T_{0}=\underline{T}=\frac{\underline{u}\left(1-e^{-\gamma \Theta_{2} / \alpha}\right)+\bar{u}\left(1-e^{-\gamma \Theta_{1} / \alpha}\right) e^{-\gamma \Theta_{2} / \alpha}}{1-e^{-\gamma \Theta / \alpha}} .
$$

It follows from (13) and (14) that

$$
x_{3}^{\Theta}\left(\Theta_{1}\right)=\frac{\bar{u}\left(1-e^{-\gamma \Theta_{1} / \alpha}\right)+\underline{u}\left(1-e^{-\gamma \Theta_{2} / \alpha}\right) e^{-\gamma \Theta_{1} / \alpha}}{1-e^{-\gamma \Theta / \alpha}} .
$$
form,

Now we set $\bar{T}=x_{3}^{\Theta}\left(\Theta_{1}\right)$ and $\hat{\gamma}=\gamma / \alpha$ and write the $\Theta$-periodic solution of Eq. (10) in closed

$$
x_{3}^{\Theta}(t)=\left\{\begin{array}{ll}
\bar{u}+(\underline{T}-\bar{u}) e^{-\hat{\gamma}(t-q \Theta)} & \text { if } t \in\left[q \Theta, q \Theta+\Theta_{1}\right) \\
\underline{u}+(\bar{T}-\underline{u}) e^{-\hat{\gamma}\left(t-q \Theta-\Theta_{1}\right)} & \text { if } t \in\left[q \Theta+\Theta_{1}, q \Theta+\Theta\right),
\end{array} \quad q=0,1,2, \ldots\right.
$$

Now assume that the parameters $\bar{u}, \underline{u}, \Theta_{1}$, and $\Theta$ of the controller (9) have been chosen so that

$$
\underline{T}<M_{1}, \quad \bar{T}>A_{2} .
$$

Assuming that the component $x_{3}$ of the solution of the closed-loop system (4), (9) satisfies the initial condition $x_{3}(0)=\underline{T}$, consider the subsystem

$$
\begin{aligned}
& \dot{x}_{1}(t)=x_{2}(t), \\
& \dot{x}_{2}(t)=-\left(\frac{k}{m}+\frac{\alpha}{l m} \varphi(t)\right) x_{1}(t)-\frac{\beta}{m} x_{2}(t)-g+\frac{\alpha}{l m} \Delta \varphi(t), \\
& x_{1}(0)=y_{0}, \quad x_{2}(0)=0
\end{aligned}
$$

where $\varphi(t)=E\left(x_{3}^{\Theta}(t)\right)$. By virtue of conditions (15) and the fact that $x_{3}^{\Theta}(t)$ is a $\Theta$-periodic solution of Eq. (10), the function $\varphi(t)$ will be $\Theta$-periodic as well, and we can write

$$
\varphi(t)= \begin{cases}E_{1}, & t \in\left[q \Theta, q \Theta+\Theta_{A_{1}}\right) \\ \frac{\bar{u}+(\underline{T}-\bar{u}) e^{-\hat{\gamma}(t-q \Theta)}-A_{1}}{A_{2}-A_{1}}\left(E_{2}-E_{1}\right)+E_{1}, & t \in\left[q \Theta+\Theta_{A_{1}}, q \Theta+\Theta_{A_{2}}\right) \\ E_{2}, & t \in\left[q \Theta+\Theta_{A_{2}}, q \Theta+\Theta_{1}\right) \\ E_{2}, & t \in\left[q \Theta+\Theta_{1}, q \Theta+\Theta_{M_{2}}\right) \\ \underline{\underline{u}+(\bar{T}-\underline{u}) e^{-\hat{\gamma}\left(t-q \Theta-\Theta_{1}\right)}-M_{1}}\left(E_{2}-E_{1}\right)+E_{1}, & t \in\left[q \Theta+\Theta_{M_{2}} q \Theta+\Theta_{M_{1}}\right) \\ M_{2}-M_{1} & t \in\left[q \Theta+\Theta_{M_{1}}, q \Theta+\Theta\right),\end{cases}
$$

where

$$
\begin{aligned}
\Theta_{A_{1}} & =\frac{1}{\hat{\gamma}} \ln \frac{\underline{T}-\bar{u}}{A_{1}-\bar{u}}, & \Theta_{A_{2}} & =\frac{1}{\hat{\gamma}} \ln \frac{\underline{T}-\bar{u}}{A_{2}-\bar{u}}, \\
\Theta_{M_{1}} & =\Theta_{1}+\frac{1}{\hat{\gamma}} \ln \frac{\bar{T}-\underline{u}}{M_{1}-\underline{u}}, & \Theta_{M_{2}} & =\Theta_{1}+\frac{1}{\hat{\gamma}} \ln \frac{\bar{T}-\underline{u}}{M_{2}-\underline{u}},
\end{aligned}
$$

and the inequality $T_{0}<M_{1}$ has been taken into account.

Figures 3 and 4 schematically depict the graphs of the functions $x_{3}^{\Theta}(t)$ and $\varphi(t)$ for $t \in[0, \Theta]$. 


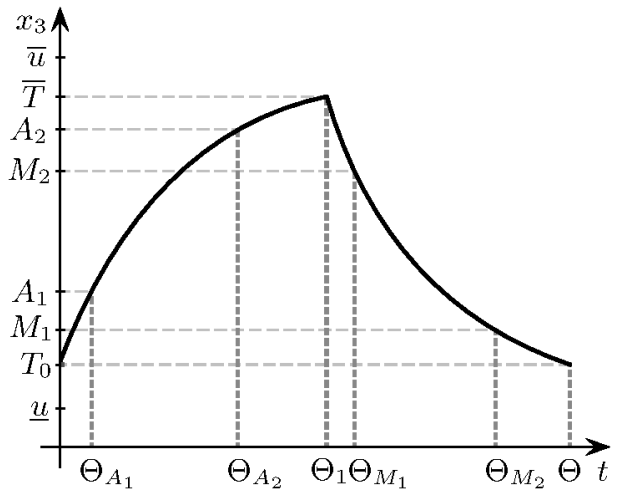

Fig. 3. Graph of the function $x_{3}^{\Theta}(t)$.

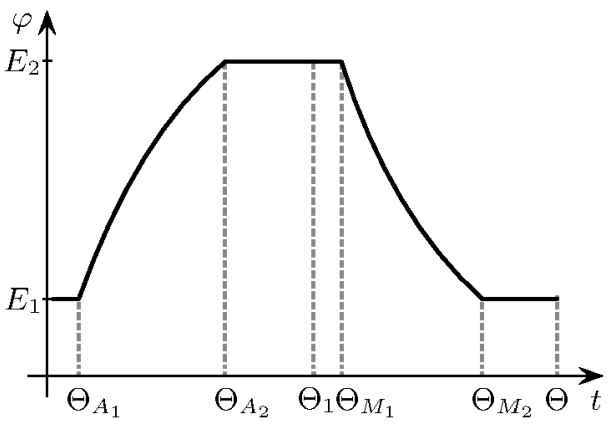

Fig. 4. Graph of the function $\varphi(t)$. form

It follows from the preceding that system (16) is actually a linear time-varying system of the

$$
\dot{x}(t)=A(t) x(t)+f(t),
$$

where $x=\left(x_{1}, x_{2}\right)^{\mathrm{T}}$ and

$$
A(t)=\left(\begin{array}{cc}
0 & 1 \\
-\frac{k}{m}-\frac{\alpha}{l m} \varphi(t) & -\frac{\beta}{m}
\end{array}\right), \quad f(t)=\left(\begin{array}{c}
0 \\
\frac{\alpha}{l m} \Delta \varphi(t)-g
\end{array}\right),
$$

the matrix $A(t)$ and the column vector $f(t)$ being continuous $\Theta$-periodic functions; i.e., in particular, $A(t+\Theta)=A(t)$ and $f(t+\Theta)=f(t)$ for each $t \geq 0$.

It is well known [5, p. 215] that if the linear homogeneous $\Theta$-periodic system

$$
\dot{x}(t)=A(t) x(t)
$$

does not have a nontrivial $\Theta$-periodic solution, then the corresponding inhomogeneous system (18) has a unique $\Theta$-periodic solution. Now note that if the homogeneous system (20) is asymptotically stable, then it cannot have a nontrivial $\Theta$-periodic solution.

Let us obtain conditions under which the linear homogeneous system (20) is asymptotically stable. To this end, we use the method proposed in [5, p. 197]. Consider system (20) with coefficient matrix $A(t)$ given by (19). This system is equivalent to the second-order differential equation

$$
\ddot{y}(t)+a \dot{y}(t)+b(t) y(t)=0,
$$

where $y(t)=x_{1}(t), \dot{y}(t)=x_{2}(t)$, and

$$
a=\frac{\beta}{m}, \quad b(t)=\frac{\alpha}{l m} \varphi(t)+\frac{k}{m} .
$$

Let us make the standard change of dependent variable $y=e^{-a t / 2} z$ in Eq. (21); then

$$
\dot{y}=\left(\dot{z}-\frac{a}{2} z\right) e^{-a t / 2} \quad \text { and } \quad \ddot{y}=\left(\ddot{z}-a \dot{z}+\frac{a^{2}}{4} z\right) e^{-a t / 2} .
$$

Therefore, this change of variable reduces Eq. (21) to the form

$$
\ddot{z}(t)+p(t) z(t)=0,
$$

where

$$
p(t)=\frac{\alpha}{l m} \varphi(t)+\frac{k}{m}-\frac{\beta^{2}}{4 m^{2}} .
$$

DIFFERENTIAL EQUATIONS $\quad$ Vol. 57 No. $8 \quad 2021$ 
According to the results in the monograph [5, p. 202], if the $\Theta$-periodic function $p(t)$ satisfies the inequalities

$$
p(t) \geq 0, \quad 0<\Theta \int_{0}^{\Theta} p(t) d t \leq 4,
$$

then all solutions $z(t)$ of Eq. (22) are bounded together with their first derivatives. However, the boundedness of $z(t)$ and $\dot{z}(t)$ implies that the solutions $y(t)$ of Eq. (21), together with their derivatives $\dot{y}(t)$, tend to zero, and consequently, system (20) is asymptotically stable. Since $E_{1} \leq \varphi(t) \leq E_{2}$, we obtain a sufficient condition for system (20) with coefficient matrix $A(t)$ given by (19) to be asymptotically stable in the form

$$
\frac{\alpha}{l m} E_{1}+\frac{k}{m}-\frac{\beta^{2}}{4 m^{2}}>0, \quad \frac{\alpha}{l m} E_{2}+\frac{k}{m}-\frac{\beta^{2}}{4 m^{2}} \leq \frac{4}{\Theta^{2}} .
$$

Consequently, the linear inhomogeneous system (18) has a unique $\Theta$-periodic solution under condition (23). We denote this solution by $x^{\Theta}(t)=\left(x_{1}^{\Theta}(t), x_{2}^{\Theta}(t)\right)^{\mathrm{T}}$.

Thus, we have proved the following assertion.

Theorem 1. Let the parameters $\bar{u}, \underline{u}, \Theta_{1}$, and $\Theta$ of the programmed control (9) for system (4) satisfy the following conditions:

1. One has the inequalities

$$
\begin{aligned}
M_{1}<\underline{T} & =\frac{\underline{u}\left(1-e^{-\gamma\left(\Theta-\Theta_{1}\right) / \alpha}\right)+\bar{u}\left(1-e^{-\gamma \Theta_{1} / \alpha}\right) e^{-\gamma\left(\Theta-\Theta_{1}\right) / \alpha}}{1-e^{-\gamma \Theta / \alpha}}, \\
A_{2}>\bar{T} & =\frac{\bar{u}\left(1-e^{-\gamma \Theta_{1} / \alpha}\right)+\underline{u}\left(1-e^{-\gamma\left(\Theta-\Theta_{1}\right) / \alpha}\right) e^{-\gamma \Theta_{1} / \alpha}}{1-e^{-\gamma \Theta / \alpha}} .
\end{aligned}
$$

2. Condition (23) holds.

Then there exists a unique periodic motion $x^{\Theta}(t)=\left(x_{1}^{\Theta}(t), x_{2}^{\Theta}(t), x_{3}^{\Theta}(t)\right)^{\mathrm{T}}$ in system $(4)$ with the programmed control (9). Furthermore, $x_{3}^{\Theta}(0)=\underline{T}$.

\section{PERIODIC MODE IN THE SYSTEM WITH A FEEDBACK}

Let us return to the original problem (see Sec. 2) of constructing a feedback control (3) ensuring the existence of a periodic mode in the closed-loop system (5). The main idea for solving this problem is to choose the parameters of the feedback (3) based on the results in the paper [1] guaranteeing the existence of an oscillatory mode in the closed-loop system (5) and also based on the programmed control (9) calculated in accordance with Theorem 1 and the periodic solution $x^{\Theta}(t)$ produced by this control.

Thus, the following assertion holds based on Theorem 1 and sufficient conditions obtained in [1] for the existence of oscillatory modes.

Theorem 2. Assume that

1. The parameters of system (4) satisfy the inequality

$$
\Delta-\frac{m g+k \Delta}{k+\alpha E_{1} / l}>0
$$

2. The spectrum of each of the matrices

$$
\left(\begin{array}{cc}
0 & 1 \\
-\frac{k l+\alpha E_{1}}{m} & -\frac{\beta}{m}
\end{array}\right), \quad\left(\begin{array}{cc}
0 & 1 \\
-\frac{k l+\alpha E_{2}}{m} & -\frac{\beta}{m}
\end{array}\right)
$$

lies on the negative half-axis and is simple. 
3. The parameters $\bar{u}, \underline{u}, \Theta_{1}$, and $\Theta$ satisfy the conditions in Theorem 1 and, in addition,

$$
\underline{u}<M_{1}, \quad \bar{u}>A_{2} .
$$

4. The numbers $y_{1}=x_{1}^{\Theta}(0)$ and $y_{2}=x_{1}^{\Theta}\left(\Theta_{1}\right)$ satisfy the inequalities

$$
y_{1}>\Delta-\frac{m g+k \Delta}{k+\alpha E_{1} / l}, \quad y_{2}<\Delta-\frac{m g+k \Delta}{k+\alpha E_{2} / l},
$$

where $x^{\Theta}(t)=\left(x_{1}^{\Theta}(t), x_{2}^{\Theta}(t), x_{3}^{\Theta}(t)\right)^{\mathrm{T}}$ is the periodic solution of system (4) supplemented by the programmed control (9) with parameters $\bar{u}, \underline{u}, \Theta_{1}$, and $\Theta$.

5. The following inequalities hold for system (4) supplemented by the feedback (3) with parameters $\bar{u}, \underline{u}, y_{1}$, and $y_{2}$ :

$$
\frac{E_{2}}{E_{1}}-\frac{\beta C_{3}^{2}}{\bar{t}_{1}^{*}}<1, \quad\left(\frac{m^{2} g^{2}}{k}+\frac{\alpha E_{2}}{l} \Delta^{2}\right) \nu_{0}<k\left(\Delta+\frac{m g}{k}\right)^{2}
$$

where

$$
\begin{gathered}
\bar{t}_{1}^{*}=\min \left\{\frac{1}{C_{2} \sqrt{2 m}},\left(y_{2}-y_{1}\right) \sqrt{\frac{E_{1} m}{2 E_{2} C_{1}}}\right\}, \\
C_{1}=k\left(y_{1}+\frac{m g}{k}\right)^{2}+\frac{\alpha}{l} E_{2}\left(y_{1}-\Delta\right)^{2}, \quad C_{2}=\frac{E_{2}}{m E_{1}}\left(\frac{\beta}{\sqrt{m}}+\sqrt{k}+\sqrt{\frac{\alpha}{l} E_{2}}\right), \\
C_{3}=\sqrt{\frac{E_{1}}{E_{2}\left(k+\alpha E_{2} / l\right)}}, \quad \nu_{0}=\max \left\{H_{\max } / H_{0}, 2\right\}, \quad H_{0}=\frac{4 m g \Delta E_{1}}{k+E_{1}}, \\
H_{\max }=\left(\frac{E_{2}}{E_{1}}\right)^{2} \bar{H}^{*}, \quad \bar{H}^{*}=\max \left\{H^{*}, \bar{H}\right\}, \quad \bar{H}=\max \left\{\frac{m^{2} g^{2}}{k}+\frac{\alpha}{l} E_{2} \Delta^{2}, k\left(\Delta+\frac{m g}{k}\right)^{2}\right\}, \\
H^{*}=\max \left\{\left(\frac{2 \beta C_{3} C_{4} / \bar{t}_{1}^{*}}{1-E_{2} / E_{1}}+\beta C_{3}^{2} / \bar{t}_{1}^{*}\right)^{2}, \frac{C_{4}^{2}}{C_{3}^{2}}\right\}, \quad C_{4}=\frac{m g}{k}+\Delta+y_{1} ;
\end{gathered}
$$

6. The conditions

$$
0<x_{1}^{\Theta}\left(t_{0}\right)<\zeta_{+}, \quad x_{2}^{\Theta}\left(t_{0}\right)=0, \quad t_{0}<\Theta_{A_{2}},
$$

are satisfied at time $t_{0}$, where $\zeta_{+}$is the positive root of the quadratic trinomial

$$
p(\zeta) \equiv k \nu_{0}\left(\zeta+\frac{m g}{k}\right)^{2}+\frac{\alpha \nu_{0} E_{2}}{l}(\zeta-\Delta)^{2}-k\left(\Delta+\frac{m g}{k}\right)^{2} .
$$

Then the solution $\tilde{x}(t)$ of the closed-loop system (5) with the initial conditions

$$
\tilde{x}_{1}(0)=x_{1}^{\Theta}\left(t_{0}\right), \quad \tilde{x}_{2}(0)=x_{2}^{\Theta}\left(t_{0}\right), \quad \tilde{x}_{3}(0)=x_{3}^{\Theta}\left(t_{0}\right)
$$

is an oscillatory mode with the parameters $t_{1}^{*}, t_{2}^{*}, y_{1}$, and $y_{2}$, where

$$
t_{1}^{*}=\left(y_{2}-y_{1}\right) \sqrt{m / H_{\max }} .
$$

(An algorithm for calculating the constant $t_{2}^{*}$ is rather awkward; it is presented in full in [1].) Further, the solution $\tilde{x}(t)$ satisfies the identity $\tilde{x}(t) \equiv x^{\Theta}\left(t+t_{0}\right)$ for $t \in\left[0, t_{1}^{*}\right]$.

Now let us show that, under certain additional conditions on the constant $t_{1}^{*}$, the oscillatory mode $\tilde{x}(t)$ of system (5) with the initial conditions (24) is actually a periodic mode. To this end, it suffices to establish that if the parameters $\bar{u}, \underline{u}, y_{1}$, and $y_{2}$ of the bang-bang feedback control (3) satisfy the assumptions of Theorem 2, then, under the initial conditions (24), the control switches occur on the interval $[0, \Theta]$ at $t_{1}=\Theta_{1}-t_{0}$ and $t_{2}=\Theta-t_{0}$. 
Thus, let the assumptions of Theorem 2 be satisfied, and let the inequality

$$
\Theta_{A_{2}}-t_{0}<t_{1}^{*} \leq \Theta_{1}-t_{0}
$$

hold for $t_{1}^{*}$. Since the solution $\tilde{x}(t)$ of the closed-loop system (5) with the initial conditions (24) is oscillatory, we have the inequality

$$
\tilde{x}_{1}(t)<y_{2}, \quad t \in\left[0, \Theta_{A_{2}}-t_{0}\right] .
$$

It follows by Theorem 2 that the identity $\tilde{x}(t) \equiv x^{\Theta}\left(t+t_{0}\right)$ holds for $t \in\left[0, \Theta_{A_{2}}-t_{0}\right]$.

Further, consider the behavior of the functions $\tilde{x}_{1}(t)$ and $\tilde{x}_{2}(t)$ on the interval $\left[\Theta_{A_{2}}-t_{0}, \Theta_{1}-t_{0}\right]$. First, note that, by the definition of the feedback (3), the identities $\tilde{x}_{1}(t) \equiv x_{1}^{\Theta}\left(t+t_{0}\right)$ and $\tilde{x}_{2}(t) \equiv x_{2}^{\Theta}\left(t+t_{0}\right)$ remain valid on the interval $\left[\Theta_{A_{2}}-t_{0}, \Theta_{1}-t_{0}\right]$ until time $t_{*} \in\left[\Theta_{A_{2}}-t_{0}, \Theta_{1}-t_{0}\right]$ such that $\tilde{x}_{1}\left(t_{*}\right)=x_{1}^{\Theta}\left(t_{*}+t_{0}\right)=y_{2}$. Let us show that $x_{1}^{\Theta}\left(t+t_{0}\right) \neq y_{2}$ for $t \in\left[\Theta_{A_{2}}-t_{0}, \Theta_{1}-t_{0}\right)$. Since $\varphi\left(t+t_{0}\right) \equiv E_{2}$ on the interval $\left[\Theta_{A_{2}}-t_{0}, \Theta_{1}-t_{0}\right]$, it follows that the functions $x_{1}^{\Theta}\left(t+t_{0}\right)$ and $x_{2}^{\Theta}\left(t+t_{0}\right)$ identically coincide on this interval with the respective components $\hat{x}_{1}(t)$ and $\hat{x}_{2}(t)$ of the solution $\hat{x}(t)$ of the linear time-invariant system

$$
\begin{aligned}
& \dot{x}_{1}(t)=x_{2}(t), \\
& \dot{x}_{2}(t)=-\left(\frac{k}{m}+\frac{\alpha}{l m} E_{2}\right) x_{1}(t)-\frac{\beta}{m} x_{2}(t)-g+\frac{\alpha}{l m} \Delta E_{2}
\end{aligned}
$$

with the initial conditions

$$
x_{1}\left(\Theta_{A_{2}}-t_{0}\right)=x_{1}^{\Theta}\left(\Theta_{A_{2}}\right), \quad x_{2}\left(\Theta_{A_{2}}-t_{0}\right)=x_{2}^{\Theta}\left(\Theta_{A_{2}}\right) .
$$

By condition 2 in Theorem 2, the coefficient matrix of system (27) is stable and has simple spectrum. Let us show that the solution $\hat{x}(t)$ of this system with the initial conditions (28) satisfies the relation $\hat{x}_{1}(t) \neq y_{2}$ for $t \in\left[\Theta_{A_{2}}-t_{0}, \Theta_{1}-t_{0}\right)$.

Indeed, the first component $x_{1}(t)$ of each solution $x(t)$ of system (27) has the property

$$
x_{1}(t) \rightarrow x_{1}^{*}>y_{2} \quad \text { as } t \rightarrow+\infty,
$$

where $x_{1}^{*}=\Delta-(m g+k \Delta) /\left(k+\alpha E_{2} / l\right)$; the convergence in (29) was proved in [1], while the inequality in (29) is the second inequality in condition 4 in Theorem 2.

Consider the equation

$$
\hat{x}_{1}(t)=y_{2}, \quad t \geq \Theta_{A_{2}}-t_{0} .
$$

Since the vector function $\left(\hat{x}_{1}(t), \hat{x}_{2}(t)\right)^{\mathrm{T}}$ on the interval $\left[\Theta_{A_{2}}-t_{0}, \Theta_{1}-t_{0}\right]$ is a solution of system (27) whose coefficient matrix has negative distinct eigenvalues $\lambda_{1}$ and $\lambda_{2}$, we have the representation

$$
\hat{x}_{1}(t)=C_{1} e^{\lambda_{1} t}+C_{2} e^{\lambda_{2} t}+x_{1}^{*},
$$

where $C_{1}$ and $C_{2}$ are some constants, which are not zero simultaneously, because

$$
\hat{x}_{1}\left(\Theta_{A_{2}}-t_{0}\right)<y_{2}<x_{1}^{*} .
$$

Therefore, Eq. (30) can be written in the form

$$
C_{1} e^{\lambda_{1} t}+C_{2} e^{\lambda_{2} t}=y_{2}-x_{1}^{*}, \quad \text { where } \quad \lambda_{1}<0, \quad \lambda_{2}<0, \quad C_{1}^{2}+C_{2}^{2}>0, \quad t>\Theta_{A_{2}} .
$$

Note that the function on the left-hand side in this equation may have at most one point of extremum for $t>0$, and hence Eq. (30) may have at most one root by virtue of property (29). Consequently, the equation $x_{1}^{\Theta}\left(t+t_{0}\right)=y_{2}$ may have at most one root on the interval $t \in\left[\Theta_{A_{2}}-t_{0}, \Theta_{1}-t_{0}\right]$. Since the function $x_{1}^{\Theta}\left(t+t_{0}\right)$ takes the value $y_{2}$ at the point $t=\Theta_{1}-t_{0}$ by a condition in Theorem 2, we conclude that $x_{1}^{\Theta}\left(t+t_{0}\right) \neq y_{2}$ for $t \in\left[\Theta_{A_{2}}-t_{0}, \Theta_{1}-t_{0}\right)$. Thus,

$$
\tilde{x}_{1}(t) \neq y_{2}, \quad t \in\left[\Theta_{A_{2}}-t_{0}, \Theta_{1}-t_{0}\right), \quad \text { and } \quad \tilde{x}_{1}\left(\Theta_{1}-t_{0}\right)=y_{2} .
$$


Now assume that the condition

$$
\Theta_{M_{1}}-\Theta_{1}<t_{1}^{*} \leq \Theta_{2}
$$

is additionally satisfied for $t_{1}^{*}$. Since the solution $\tilde{x}(t)$ of the closed-loop system (5) with the initial conditions (24) is an oscillatory mode, we have the inequality $\tilde{x}_{1}(t)>y_{1}, \quad t \in\left[\Theta_{1}-t_{0}, \Theta_{M_{1}}-t_{0}\right]$. It follows in view of relations (31) and (26) that

$$
\tilde{x}(t) \equiv x^{\Theta}\left(t+t_{0}\right) \quad \text { for } \quad t \in\left[0, \Theta_{M_{1}}-t_{0}\right] .
$$

Now consider the behavior of the functions $\tilde{x}_{1}(t)$ and $\tilde{x}_{2}(t)$ on the interval $\left[\Theta_{M_{1}}-t_{0}, \Theta-t_{0}\right]$. By analogy with the preceding argument, note that the identities $\tilde{x}_{1}(t) \equiv x_{1}^{\Theta}\left(t+t_{0}\right)$ and $\tilde{x}_{2}(t) \equiv x_{2}^{\Theta}\left(t+t_{0}\right)$ remain valid on the interval $\left[\Theta_{M_{1}}-t_{0}, \Theta-t_{0}\right]$ until time $t_{*} \in\left[\Theta_{M_{1}}-t_{0}, \Theta-t_{0}\right]$ such that $\tilde{x}_{1}\left(t_{*}\right)=x_{1}^{\Theta}\left(t_{*}+t_{0}\right)=y_{1}$. Let us show that $x_{1}^{\Theta}\left(t+t_{0}\right) \neq y_{1}$ for $t \in\left[\Theta_{M_{1}}-t_{0}, \Theta-t_{0}\right)$. Since $\varphi\left(t+t_{0}\right) \equiv E_{1}$ on the interval $\left[\Theta_{M_{1}}-t_{0}, \Theta-t_{0}\right]$, it follows that the functions $x_{1}^{\Theta}\left(t+t_{0}\right)$ and $x_{2}^{\Theta}\left(t+t_{0}\right)$ identically coincide on this interval with the respective components $\hat{x}_{1}(t)$ and $\hat{x}_{2}(t)$ of the solution $\hat{x}(t)$ of the linear time-invariant system

$$
\begin{aligned}
& \dot{x}_{1}(t)=x_{2}(t) \\
& \dot{x}_{2}(t)=-\left(\frac{k}{m}+\frac{\alpha}{l m} E_{1}\right) x_{1}(t)-\frac{\beta}{m} x_{2}(t)-g+\frac{\alpha}{l m} \Delta E_{1}
\end{aligned}
$$

with the initial conditions

$$
x_{1}\left(\Theta_{M_{1}}-t_{0}\right)=x_{1}^{\Theta}\left(\Theta_{M_{1}}\right), \quad x_{2}\left(\Theta_{M_{1}}-t_{0}\right)=x_{2}^{\Theta}\left(\Theta_{M_{1}}\right) .
$$

By condition 2 in Theorem 2, the coefficient matrix of system (34) is stable and has simple spectrum. Let us show that the solution $\hat{x}(t)$ of this system with the initial conditions (35) satisfies the relation $\hat{x}_{1}(t) \neq y_{1}$ for $t \in\left[\Theta_{M_{1}}-t_{0}, \Theta-t_{0}\right)$.

Indeed, the first component $x_{1}(t)$ of each solution $x(t)$ of system (34) has the property

$$
x_{1}(t) \rightarrow x_{1}^{* *}<y_{1} \quad \text { as } t \rightarrow \infty,
$$

where $x^{* *}=\Delta-(m g+k \Delta) /\left(k+\alpha E_{1} / l\right)$; the convergence in (36) was proved in the paper [1], while the inequality is the first inequality in condition 4 in Theorem 2.

Consider the equation

$$
\hat{x}_{1}(t)=y_{1}, \quad t \geq \Theta_{M_{1}}-t_{0} .
$$

Since the vector function $\left(x_{1}^{\Theta}(t), x_{2}^{\Theta}(t)\right)^{\mathrm{T}}$ on the interval $\left[\Theta_{M_{1}}-t_{0}, \Theta-t_{0}\right]$ is a solution of system (34) whose coefficient matrix has distinct negative eigenvalues $\lambda_{1}^{\prime}$ and $\lambda_{2}^{\prime}$, one has the representation

$$
\hat{x}_{1}(t)=C_{1} e^{\lambda_{1}^{\prime} t}+C_{2} e^{\lambda_{2}^{\prime} t}+x_{1}^{* *},
$$

where $C_{1}$ and $C_{2}$ are some constants, which are not zero simultaneously, because

$$
\hat{x}_{1}\left(\Theta_{M_{1}}-t_{0}\right)>y_{1}>x_{1}^{* *} .
$$

Therefore, Eq. (37) can be written in the form

$$
C_{1} e^{\lambda_{1}^{\prime} t}+C_{2} e^{\lambda_{2}^{\prime} t}=y_{1}-x_{1}^{* *}, \quad \text { where } \quad \lambda_{1}^{\prime}<0, \quad \lambda_{2}^{\prime}<0, \quad C_{1}^{2}+C_{2}^{2}>0, \quad t \geq \Theta_{M_{1}}-t_{0} .
$$

Note that the function on the left-hand side in this equation may have at most one point of extremum for $t>0$, and then Eq. (37) may have at most one root by virtue of property (36). Consequently, the equation $x_{1}^{\Theta}\left(t+t_{0}\right)=y_{1}$ may have at most one root on the interval $t \in\left[\Theta_{M_{1}}-t_{0}, \Theta-t_{0}\right]$. Since the function $x_{1}^{\Theta}\left(t+t_{0}\right)$ takes the value $y_{1}$ at the point $t=\Theta-t_{0}$ by a condition in Theorem 2, we conclude that $x_{1}^{\Theta}\left(t+t_{0}\right) \neq y_{1}$ for $t \in\left[\Theta_{M_{1}}-t_{0}, \Theta-t_{0}\right)$. Thus,

$$
\tilde{x}_{1}(t) \neq y_{1}, \quad t \in\left[\Theta_{M_{1}}-t_{0}, \Theta-t_{0}\right), \quad \text { and } \quad \tilde{x}_{1}\left(\Theta-t_{0}\right)=y_{1} .
$$

DIFFERENTIAL EQUATIONS $\quad$ Vol. $57 \quad$ No. $8 \quad 2021$ 
This, together with identity (33), implies that

$$
\tilde{x}(t) \equiv x^{\Theta}\left(t+t_{0}\right) \quad \text { for } \quad t \in\left[0, \Theta-t_{0}\right] .
$$

Let us impose one more constraint on the constant $t_{1}^{*}$; namely, let

$$
t_{1}^{*}>t_{0}
$$

Since the solution $\tilde{x}(t)$ of the closed-loop system (5) with the initial conditions (24) is an oscillatory mode, it follows that

$$
\tilde{x}_{1}(t)<y_{2}, \quad t \in\left[\Theta-t_{0}, \Theta\right] .
$$

This, together with identity (38), implies that the switching times of the bang-bang control (3) and the programmed control (9) for $t \in[0, \Theta]$ coincide. Then one has the identity $\tilde{x}(t) \equiv x^{\Theta}\left(t+t_{0}\right)$ on this interval, and this identity remains valid for all $t \geq 0$ by virtue of the $\Theta$-periodicity of the function $x^{\Theta}\left(t+t_{0}\right)$.

Consequently, the oscillatory mode $\tilde{x}(t)$ of system (5) with the initial conditions (24) is a periodic mode. Thus, we have proved the following assertion about the existence of a periodic mode in the closed-loop system (5).

Theorem 3. Let the assumptions of Theorem 2 be satisfied, and let inequalities (25), (32), and (39) hold. Then there exists a periodic mode with the initial conditions (24) in the closed-loop system (5).

\section{SIMULATION RESULTS}

Consider system (1) with the parameters

$$
\begin{gathered}
m=0.018, \quad \beta=0.7, \quad k=0.07, \quad \alpha=1.32 \cdot 10^{-8}, \quad l=0.2, \quad \Delta=0.009, \quad \gamma=1.76 \cdot 10^{9}, \\
M_{1}=24.3, \quad M_{2}=52.7, \quad A_{1}=32.8, \quad A_{2}=57.5, \quad E_{1}=3 \cdot 10^{8}, \quad E_{2}=8 \cdot 10^{8} .
\end{gathered}
$$

Using the results in the present paper, we can calculate the parameters

$$
\bar{u}=80, \quad \underline{u}=1, \quad y_{1}=0.00172, \quad y_{2}=0.0063
$$

of the feedback (3) for which there exists a periodic motion (Fig. 5) with period $\Theta=0.2$ in the corresponding closed-loop system with $E(0)=4.73 \cdot 10^{8}$ and with the initial conditions

$$
y(0)=0.0032, \quad \dot{y}(0)=0, \quad T(0)=41.067 .
$$

Here the bang-bang control switching times are $t_{1}=\Theta_{1}-t_{0}$ and $t_{2}=\Theta-t_{0}$, where $\Theta_{1}=0.1$ and $t_{0}=0.036$.

\section{CONCLUSIONS}

The paper considers a mathematical model of a thermal energy harvester [1] in the form of the controlled nonlinear dynamical system (1) whose coefficients are positive numerical parameters. The main problem is to choose a control algorithm ensuring the occurrence of a periodic motion in the closed-loop system. As a control algorithm, it is proposed to use output feedback with feedback operator in the form of a bang-bang hysteresis control. As a result, sufficient conditions are obtained for the coefficients and initial values of the state variables of system (1) and for the parameters of the controller (3) ensuring the occurrence of a periodic mode in the closed-loop system.

\section{FUNDING}

This work was supported by the Russian Foundation for Basic Research and the National Science Foundation of Bulgaria, project no. 19-57-18006; the Russian Foundation for Basic Research, projects nos. 20-57-0001, 20-07-00827, and 19-07-00294; and the RF Ministry of Education and Science within the framework of the program of the Moscow Center for Fundamental and Applied Mathematics by agreement no. 075-15-2019-1621. 


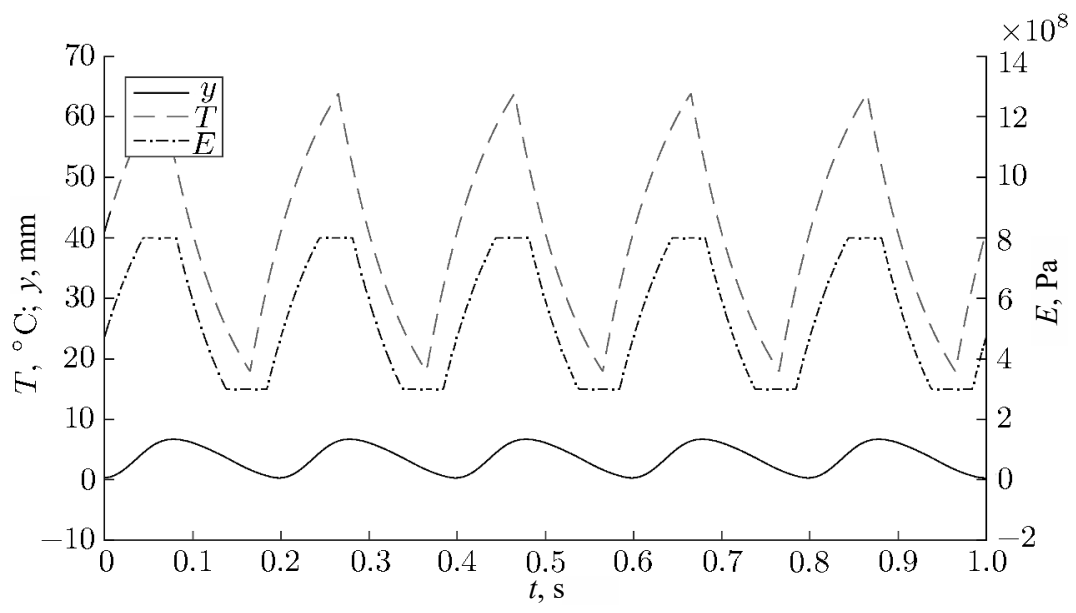

Fig. 5. Graphs of the functions $y(t), T(t)$, and $E(t)$ corresponding to a periodic mode of system (1).

\section{OPEN ACCESS}

This article is licensed under a Creative Commons Attribution 4.0 International License, which permits use, sharing, adaptation, distribution and reproduction in any medium or format, as long as you give appropriate credit to the original author(s) and the source, provide a link to the Creative Commons license, and indicate if changes were made. The images or other third party material in this article are included in the article's Creative Commons license, unless indicated otherwise in a credit line to the material. If material is not included in the article's Creative Commons license and your intended use is not permitted by statutory regulation or exceeds the permitted use, you will need to obtain permission directly from the copyright holder. To view a copy of this license, visit http://creativecommons.org/licenses/by/4.0/.

\section{REFERENCES}

1. Fursov, A.S., Todorov, T.S., Krylov, P.A., and Mitrev, R.P., On the existence of oscillatory modes in a nonlinear system with hystereses, Differ. Equations, 2020, vol. 56, no. 8, pp. 1081-1099.

2. Todorov, T., Nikolov, N., Todorov, G., and Ralev, Y., Modelling and investigation of a hybrid thermal energy harvester, MATEC Web Conf., 2018, vol. 148, p. 12002.

3. Pai, A., A phenomenological model of shape memory alloys including time-varying stress, Master Appl. Sci. Thesis, Waterloo, Ontario, Canada: Univ. Waterloo, 2007.

4. Obmorshev, A.N., Vvedenie $v$ teoriyu kolebanii (Introduction to Vibration Theory), Moscow: Nauka, 1965.

5. Demidovich, B.P., Lektsii po matematicheskoi teorii ustoichivosti (Lectures on the Mathematical Theory of Stability), Moscow: Nauka, 1967. 